\title{
New magnetic and structural results for uniformly spaced, alternatingly spaced, and ladder-like copper (II) linear chain compounds
}

\author{
William E. Hatfield \\ Department of Chemistry, University of North Carolina, Chapel Hill, North Carolina 27514
}

Results of recent synthetic, structural, and magnetic work on exchange coupled copper (II) ions bridged by nonmagnetic ligands are discussed, including the quasi-linear chain compound bis (dimethyldithiocarbamato) copper (II) in which the copper (II) ions are antiferromagnetically exchange coupled. An unusual correlation exists in the structural and magnetic data for $\left[\mathrm{Cu}(\mathrm{dmtc})_{2}\right]_{\infty}$ and other known sulfur bridged copper compounds. Data are presented for the alternatingly spaced linear chain compounds di- $\mu$-chlorobis-44methylpyridine)copper (II), and a series of dionebis-(thiosemicarbazato)copper (II) compounds. Exchange coupling constants and alternation parameters were obtained from expressions which were empirically generated from calculations on rings of ten $S=1 / 2$ ions for $k T /|J| \gtrsim 0.5$ and $J<0$. Finally, new results for recently discovered spin ladder systems are presented.

PACS numbers: $75.40 . \mathrm{Fa}, 75.30 . \mathrm{Kz}, 75.30 . \mathrm{Et}$

\section{INTRODUCT ION}

The cluster approach as developed by Bonner and Fisher (1) in their classical work on the Heisenberg chaln with $S=1 / 2$, has received much less attention in applications to exchange coupled systems with lower symmetry geometries. This is a surprising situation since the results of Bonner and Fisher have been used extensively by chemists and physicists for the analysis of magnetic properties of exchange coupled low-dimensional $S=1 / 2$ chains. A limited number of studies have been carried out. Weng followed Bonner and Fisher's cluster approach in his calculation of the magnetic properties of Heisenberg chains with $S>1$, (2) but this work has not been widely applied since exchange coupled chains with $S>1 / 2$ frequently show pronounced anisotropy in the exchange, and one of the anisotropic models, the Ising chain or the XY-chain is required for the analysis of the data.

Bonner and coworkers $(3,4)$ have continued investigating the application of the cluster approach to more complicated spin systems. These include the alternatingly spaced Heisenberg chain and the interesting spin ladder systems. It is interesting to know that the most thoroughly studied transitton metal example of an alternatingly spaced Heisenberg antiferromagnet is $\mathrm{Cu}\left(\mathrm{NO}_{3}\right)_{2} \cdot 2.5 \mathrm{H}_{2} \mathrm{O}$, a compound which has a ladder-1ike structure at room temperature. $(5,6)$ The initial attempts to analyze the magnetic data for $\mathrm{Cu}\left(\mathrm{NO}_{3}\right)_{2}$. $2.5 \mathrm{H}_{2} \mathrm{O}$ were in terms of the spin ladder. This is a very good example of a system which exhibits a structure at htgh temperature which is inconsistent with the observed magnetic properties at low temperatures. There are now several bona fide examples of alternatingly spaced He1senberg exchange coupled $S=1 / 2$ linear chains. These include the pyrazine-bridged binuclear copper acetate cha in, (7) $\left[\mathrm{Cu}_{2}(\mathrm{OAc})_{4} \mathrm{pyr}\right]_{\infty}$, the low temperature from of tetra thio fulvalenium bis-cis (1, 2-perfluoromethylethylene1,2-dithiolato) copper(II) (8) which results from a spin Peier1s transition at $12 \mathrm{k}$, catena-di- $\mu$-bromo-(Nmethylimidazole) copper(II) (9), and a series of diketobis (thiosemicarbazato) copper(II) complexes related to the carcinostatic and carcinolytic agent 3-ethoxy-2oxobutraldehyde-bis (thio sem Icarbaza to) copper(II), Cu-KTS. (10) The magnetic properties of several of these alternatingly spaced Heisenberg exchange-coupled $S=1 / 2$ linear chains have been characterized in our laboratory. (11) Basically, because of the general interest in alternating Heisenberg antiferromagnets, and because of the absence of convenient procedures for the analysis of the magnetic properties, we have repeated the calculations reported by Duffy and Barr (12) for such a model system to obtain numerical data for the generation of an expression for magnetic susceptibility in terms of the exchange coupling constant and the alternation parameter. The generation of the magnetic susceptibility expression and the application of the new expression for the analysis of new magnetic data for several of these compounds will be discussed. Since bona fide examples $S=1 / 2$ ladder systems have not been avallable for study, there has been little stimulation for theoretical work. However, in recent years, three examples of copper compounds with ladder structures have been reported. These include catena-dibromo (2-aminomethy 1)-pyridine-copper(II), (13) catena-dibromo (2-methyl-1, 2-diaminopropane) copper(II), (13) and catena-trichlorohydraziniumcopper(II) (14). Weller (15) has carried out calculations of the magnetic properties of ladder systems with a range of exchange coupling constants, and has analyzed the magnetic susceptibility for the known $\mathrm{S}=1 / 2$ ladder systems. Preliminary results of these studies on catena-trichlorohydraziniumcopper(II) are presented here.

\section{THE LINEAR CHAIN MODEL}

For an infinite linear chain the Hamiltonian may be written as

$$
\mathrm{H}=-2 \mathrm{~J} \sum_{i=1}^{\mathrm{n}}\left\{\alpha \mathrm{S}_{1+1}^{z} \mathrm{~s}_{i+1}^{z}+\gamma\left(\mathrm{s}_{i} \mathrm{x}_{+\mathrm{S}_{1+1}}^{\mathrm{x}}+\mathrm{S}_{i} \mathrm{y}_{\mathrm{S}_{i+1}}^{\mathrm{y}}\right)\right\}-\mathrm{gBH} \sum_{i=1}^{\mathrm{n} H \cdot \mathrm{S}_{1}}
$$

If $\alpha=1$ and $\gamma=1$, the 1sotroplc Heisenberg model is produced. If $\alpha=1$ and $\gamma=0$, the anisotropic Ising model is obtained, and if $\alpha=0$ and $\gamma=1$, the anisotropic $X-Y$ model results. Copper(II) systems are usually best described by the Heisenberg model, but in some cases $\gamma$-values slightly less than one have been deduced from experimental data.

In 1964, Bonner and Fisher (1) carried out calculations on systems of chains containing from 4 to 12 spins. The results for the 11 and 12 membered chains were used to extrapolate to the infinitelength chain with very good agreement above $\mathrm{kT} /|\mathrm{J}|$ of 0.5 . The maximum in susceptibility is uniquely defined by the following relations:

$$
\frac{\mathrm{kT}_{\max }}{|\mathrm{J}|}=1.282 \text { and } \frac{\chi_{\max }|\mathrm{J}|}{\mathrm{Ng}^{2} \mu_{\mathrm{B}}^{2}}=0.07346 \text {. }
$$

Hall (16) noted that the shape of the reduced magnetic susceptibility versus temperature curve obtalned by Bonner is very simflar to a serpentine curve, and $f 1 t$ the quadratic/cubic function 


$$
f(x)=\left(A x^{2}+B x+C\right) /\left(x^{3}+D x^{2}+E x+F\right)
$$

to the theoretically calculated results tabulated by Bonner. (17) A fit, with the greatest deviation between the calculated values for reduced magnetic susceptibility and those obtained by Bonner being less than $0.5 \%$, was obtained with the parameters $\mathrm{A}=0.25, \mathrm{~B}=$ $0.14995, C=0.30094, D=1.9862, E=0.68854$, and $F=6.0626$. The magnetic susceptibility of chains of isotropically exchange coupled $S=1 / 2$ ions is

$$
x_{m}=\frac{\mathrm{Ng}^{2} \mathrm{H}_{\mathrm{B}}^{2}}{\mathrm{kT}} \cdot \frac{\mathrm{A}+\mathrm{Bx}^{-1}+\mathrm{Cx}^{-2}}{1+\mathrm{Dx}^{-1}+\mathrm{Ex}^{-2}+\mathrm{Fx}^{-3}} .
$$

The maximum in reduced susceptibility calculated from this expression occurs at

$$
\frac{\mathrm{kT}_{\max }}{|\mathrm{J}|}=1.297 \text { and } \frac{x_{\max }|\mathrm{J}|}{\mathrm{Ng}^{2}{ }_{\mathrm{B}}{ }^{2}}=0.07337 .
$$

The agreement with the values of Bonner and Fisher (1) which are given above are excellent, and this expression allows data analysis using computer programs rather than graphical methods.

MAGNETIC SUSCEPTIBILITY OF THE LINEAR CHAIN BIS (DIMETHYLDITHIOCARBAMATO) COPPER(II)

Magnetic susceptibility data (18) for a powdered sample of the linear chain compound bis (dimethyldithiocarbamato) copper(II) is plotted as a function of temperature in Figure 1. It may be seen that the susceptibility exhibits a maximum value at about $2.5 \mathrm{~K}$. This behavior of the magnetic susceptibility is indicative of a magnet1cally condensed substance, and since the compound has a linear chain structure, (19) the data were initially analyzed using the Heisenberg 1 inear chain theory of Bonner and Fisher, (1) and the analytical expression developed by Ha11. (16) Since the chains in $\left[\mathrm{Cu}(\mathrm{dmtc})_{2}\right]_{\infty}$ are in rather close contact, substantial interchain interactions were anticipated. The problem was treated as one involving Helsenberg interactions along a chain with a correction for interchain interactions (19a) being accounted for in the expression

$$
x_{m}=x_{H} /\left(1-2 z J^{\prime} x_{H} / \mathrm{Ng}^{2} \mu_{B}^{2}\right)
$$

where $x_{H}$ is the magnetic susceptibility of an isolated Heisenberg chain of $S=1 / 2$ ions, $z$ is the number of near neighbors in adjacent chains, and $J^{\prime}$ is the interchain exchange parameter. For the case of $\left[\mathrm{Cu}(\mathrm{dmtc}){ }_{2}\right]_{\infty}$, $z$ was taken to be 4 . The magnetic susceptibility expression thus modified was fit to the experimental magnetic susceptibility data, and the best fit yielded the $\langle g\rangle$ value of $2.03, J=-1.22 \mathrm{~cm}^{-1}$, and $J^{\prime}=+0.47 \mathrm{~cm}^{-1}$. Since the ratio of $\mathrm{J} / \mathrm{J}$ is relatively large, the applicability of the linear chain model to this compound is open to some question. The expression for two exchangecoupled $S=1 / 2$ ions gives a reasonable fit to the data also, with the EPR $\langle g\rangle$ value of 2.03 and $\mathrm{J}=-1.3 \mathrm{~cm}^{-1}$. However, this latter model is probably not applicable to the present case since the high temperature structure is known to be a uniformly spaced linear chain, and since the magnetic susceptibility data appear to be tending to non-zero values as the temperature approaches zero. Also, there is no anomaly in the dielectric constant versus temperature plot over the temperature range $10-300 \mathrm{~K}$, and structural phase transitions are not indicated. Extensive measurements on single crystals in the low temperature range will be required before an exact description of the magnetic interaction may be had. However, for the purposes of this work, it is clear that the sulfur-bridged copper lons are exchanged coupled. Furthermore, the data are

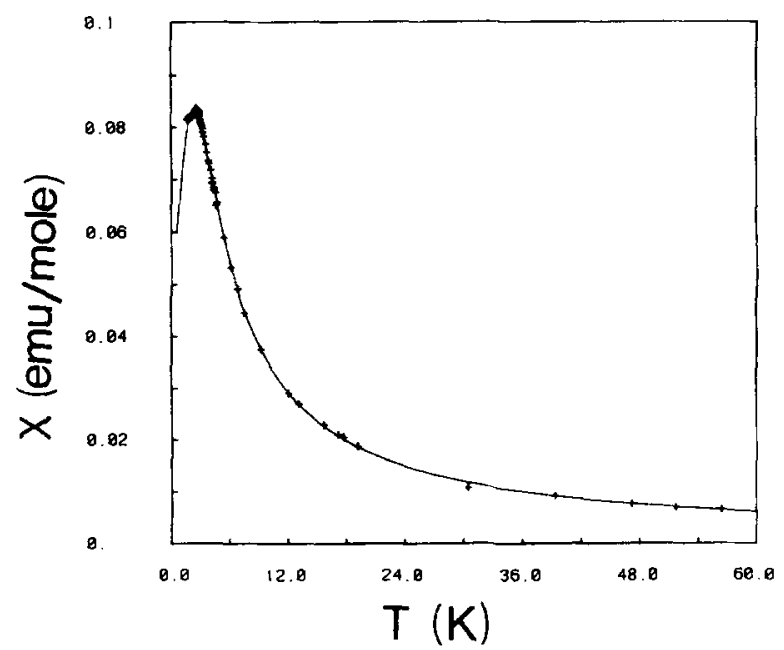

Figure 2. The magnetic susceptibility versus temperature for the linear chain compound [Cu(dntc) ${ }_{2}$. The solid line is the best fit of the Heisenberg linear chain $S=2 / 2$ model to the data with the EPR $g$ value of 2.03 and Jintrachain) $=-2.22 \mathrm{~cm}^{-2}$. A correction for an interchain interaction gave $J($ interchain $)=0.47 \mathrm{~cm}^{-2}$.

adequate to permit a correlation between the magnetic properties of this sulfur bridged chain and the geometry of the bridged $\mathrm{Cu}_{2} \mathrm{~S}_{2}$ units in a number of copper com-

plexes. The available structural and magnetic data for the sulfur-bridged copper(II) compounds are compiled in Table $I$. There are several conclusions which may be drawn from the data. The first and most important point is that even long out - f-plane bridging bond distances provide pathways for appreclable singlet-triplet splittings resulting from superexchange coupling. Next, the short in-plane copper-sulfur bond distances which are involved in the bridge are relatively constant at 2.3 $\pm 0.04 \mathrm{~A}$, but the out-of-plane bond distances vary from $2.851 \AA$ in $\left[\mathrm{Cu}(\operatorname{detc})_{2}\right]_{2}$ to $3.3 \AA$ in $\left[\mathrm{Cu}\left(\mathrm{H}^{+} \mathrm{TCH}\right) \mathrm{Cl}_{2}\right]_{2} \mathrm{Cl}_{2}$. In addition, there is a fairly wide range of $\mathrm{Cu}-\mathrm{S}_{b}-\mathrm{Cu}$ angles at the bridging sulfur atom.

Studies on a series of di- $\mu$-hydroxo-bridged copper (II) compounds have established that the singlet-triplet splitting is a linear function of the angle at the bridging oxygen atom. (28) Also, Roundhill, et al. (29) have noted that the singlet states are stablized as the bridge angle increases in a number of chlorobridged copper(II) systems. For the purposes of the present study we note that in this particular group of sulfur-bridged compounds there is an additional variable. which also arises in several parallel planar chlorobridged copper compounds, and that is the out-of-plane bridging bond distances. Since the extent of exchange coupling is known to decrease as the intranuclear separation of exchange coupling sites increases, we were led to investigate the relationship between the exchange coupling constants for these sulfur bridged compounds and the function $\phi / \mathrm{r}_{\mathrm{o}}$, where $\phi$ is the $\mathrm{Cu}-\mathrm{S}_{\mathrm{b}}-\mathrm{Cu}$ angle and $r_{0}$ is the out-of-plane copper-sulfur bond distance. This relationship is shown in Figure 2, where it may be seen that a smooth curve may be drawn through the experimental exchange coupling constants $J$ and the function $\phi / r_{0}$.

For the purposes of this work the exchange coupling constant for [Cu(detc) $\left.{ }_{2}\right]_{2}$ determined earlier in our laboratory was used although there may be some question concerning the magnitude of this number in yiew of the EPR investigations of Al'tshuler, et al. (22).

Also, the geometry of the more tightly bound unit of $[\mathrm{Cu}(\mathrm{KTS})]_{\infty}$ was used to obtain the value of $\phi / \varsigma_{0}$ for 
Table I. Structural and Magnetlc Data in SulfurBridged Copper(II) Compounds

\begin{tabular}{|c|c|c|c|c|}
\hline Compound & $\mathrm{J}, \mathrm{cm}^{-1}$ & $\begin{array}{l}\mathrm{Cu}-\mathrm{S}_{b}{ }^{a} \\
(\mathrm{~A})\end{array}$ & $\begin{array}{l}\mathrm{Cu}-\mathrm{S}-\mathrm{Cu} \\
(\mathrm{deg})\end{array}$ & $\phi / r_{0}$ \\
\hline $\begin{array}{l}{\left[\mathrm{Cu}(\text { dmd tc })_{2}\right]_{\infty}} \\
{\left[\mathrm{Cu}(\text { detc })_{2}\right]_{2}}\end{array}$ & $\begin{array}{c}-1.22^{\mathrm{b}} \\
12^{\mathrm{d}} \\
6.5 \pm 0.5^{\mathrm{f}}\end{array}$ & $\begin{array}{l}3.159(2) \\
2.851\end{array}$ & $\begin{array}{l}94.2^{\circ} \\
86.9^{\circ}\end{array}$ & $\begin{array}{l}29.8^{c} \\
30.5^{c}\end{array}$ \\
\hline $\begin{array}{l}{\left[\mathrm{Cu}\left(\mathrm{H}^{+}-\mathrm{TCH}\right) \mathrm{Cl}_{2}\right]_{2} \mathrm{Cl}_{2}} \\
{[\mathrm{Cu}(\mathrm{KTS})]_{\infty}}\end{array}$ & $\begin{array}{r}-12.41^{\mathrm{g}} \\
-8^{1}\end{array}$ & $\begin{array}{l}3.310(6) \\
3.101 \\
3.312\end{array}$ & $\begin{array}{l}88.4^{\circ} \\
89.8^{\circ} \\
86.5^{\circ}\end{array}$ & $\begin{array}{l}26.7^{\mathrm{h}} \\
29.0^{\mathrm{j}}\end{array}$ \\
\hline$\left[\mathrm{Cu}(\mathrm{d}-\mathfrak{n}-\mathrm{btc})_{2}\right]_{2}$ & $-14 \cdot 1^{k}$ & 2.899 & $92.1^{\circ}$ & $31.8^{k}$ \\
\hline
\end{tabular}

athis is the long, oyt-of-plane bridging bond distance;

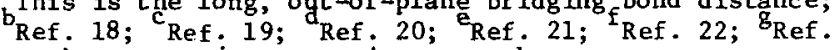

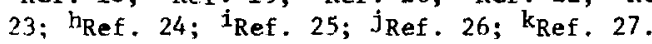

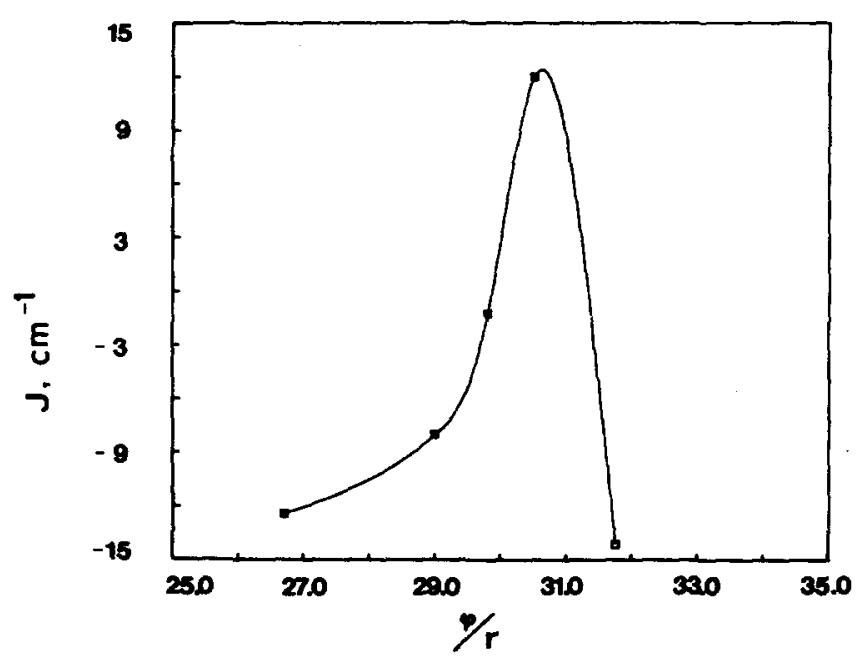

Figure 2. A plot of $J$ versus $\phi / r_{0}$, where $\phi$ is the $\mathrm{Cu}_{-} \mathrm{S}_{\mathrm{b}}$-Cu bridging angle in a series of sulfur bridged copper compounds and $r_{0}$ is the long out-of-plane bond distance.

this alternating chain compound, since this unit may be expected to dominate exchange coupling in the range of Blumberg and Peisach's measurements. (25) It is interesting to point out that at the time that the work on $\left[\mathrm{Cu}(\mathrm{dmtc})_{2}\right]_{\infty}$ was carried out, (18) the data for $\left[\mathrm{Cu}(\alpha-\mathrm{d}-\mathrm{n}-\mathrm{btc})_{2}\right]_{2}$ were not available. At that time it was expected that the line in Figure 2 would reach a maximum at some larger value of $\phi / r_{0}$ and then begin to decrease with an increase in $\phi / \mathrm{r}_{0}$. This expectation was anticipated in view of data for chloro-bridged copper(II) complexes. (30)

\section{THE ALTERNATING CHAIN MODEL}

The Hamiltonian for the Helsenberg alternating linear chains may be written as

$$
H=-2 J \sum_{i=1}^{n / 2}\left[\hat{S}_{2 i} \cdot \hat{S}_{2 i-1}+\alpha \hat{S}_{2 i} \cdot \hat{S}_{2 i+1}\right]
$$

where $J$ is the exchange integral between a spin and its $r$ ight neighbor and $\alpha J$ is the exchange integral between a spin and its left neighbor. The model of most interest is for antiferromagnet Ic exchange ( $J$ less than 0 ) and for $0 \leq \alpha \leq 1$. At the extremes, when $\alpha=0$, the model reduces to the dimer model with pairwise interactions, and when $\alpha=1$, the model reduces to the regular linear chain model.
This model has been studied in detall by Duffy and Barr, (12) as well as Bonner and Friedberg, $(3,4)$ Diederix, et al. (5), and Bonner and coworkers (30a). For the limiting case of $a=1$, Hall (16) has fit Bonner's numerical data (17) to expression (2) given above. For the purposes of this work the reduced magnetic susceptibilities of short alternating rings of up to ten $S=1 / 2$ spins for $\alpha=0,0.1,0.2$, $0.3,0.4,0.6,0.8$, and 1.0 have been calculated using the cluster approach and the programs which have been described earlier. (15,31) The results of these calculations are in excellent agreement with the previously reported results of Duffy and Barr, (12) and the reduced magnetic susceptibilities are displayed as a function of reduced temperature in Figure 3.

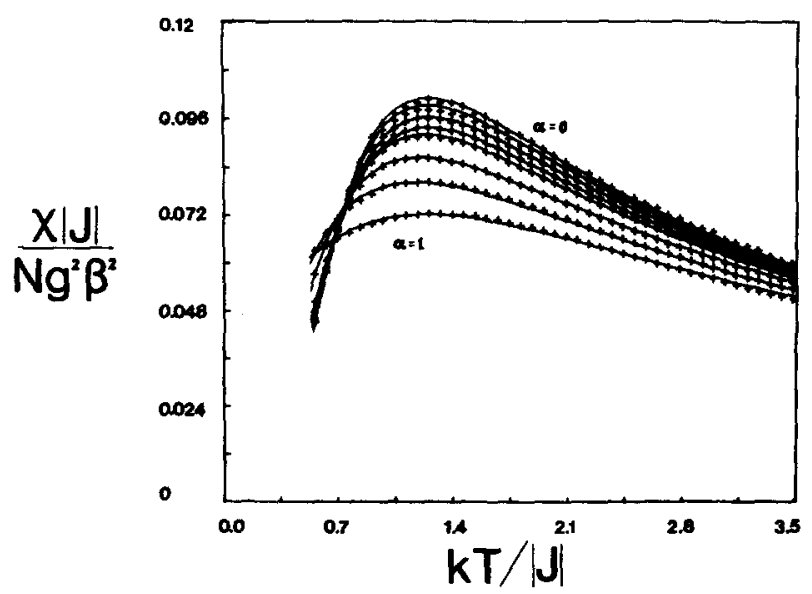

Figure 3. Plot of equation 5 (solid line) in reduced coordinates using coefficients listed in text. Points for 20 membered rings (t) are calculated with $H=2000$ oe, $g=2.2$, and $\alpha=0.0,0.2,0.2,0.3,0.4$, $0.6,0.8$, and 2.0 .

There reduced magnetic susceptibllity curves for 10 member alternating chains with the $\alpha$ values indicated above were fitted to the quadratic-cubic function (5), where the reduced susceptibility $x_{r}=x|J| / \mathrm{Ng}^{2} \mu_{B}{ }^{2}$ and the reduced temperature $T_{r}$ is gtven by $k T /|\mathrm{J}|$.

$$
x_{r}=\frac{A T_{r}^{2}+B T_{r}+C}{T_{r}^{3}+D T_{r}^{2}+E T_{r}+F}
$$

The fits were truncated below $\mathrm{kT} /|\mathrm{J}|$ of 0.5 , and the constant $A$ in expression 5 was set equal to 0.25 for convergence to the Curie Law at high temperatures. With the expressions for reduced susceptibility for each of the eight alternating chain cases as a starting point, a unified expression for $x_{r}$ as a function of the alternating parameter was developed. The values for the parameters $A, B, C, D, E$, and $F$ for $0 \leq \alpha \leq 0.4$ are

$$
\begin{aligned}
& A=0.25 \\
& B=-0.12587+0.22752 \alpha \\
& C=0.019111-0.13307 \alpha+0.509 \alpha^{2}-1.3167 \alpha^{3}+1.0081 \alpha^{4} \\
& D=0.10772+1.4192 \alpha \\
& E=-0.0028521-0.42346 \alpha+2.1953 \alpha^{2}-0.82412 \alpha^{3} \\
& F=0.37754-0.067022 \alpha+5.9805 \alpha^{2}-2.1678 \alpha^{3}+15.838 \alpha^{4}
\end{aligned}
$$

The values for the parameters $A-F$ for $0.4<\alpha \leq 1.0$ are

$$
\begin{aligned}
A= & 0.25 \\
B= & -0.13695+0.26387 \alpha \\
C= & 0.017025-0.12668 \alpha+0.49113 \alpha^{2}-1.1977 \alpha^{3} \\
& +0.87257 \alpha^{4}
\end{aligned}
$$


$D=0.070509+1.3042 \alpha$

$E=-0.0035767-0.40837 \alpha+3.4862 \alpha^{2}-0.73888 \alpha^{3}$

$F=0.36184-0.065528 \alpha+6.65875 \alpha^{2}-20.945 \alpha^{3}+$

The expression for the reduced magnetic susceptibility was then converted to magnetic susceptibility in familiar coordinates to give expresston 6

$$
x_{m}=\frac{\mathrm{Ng}^{2} \mu_{B}^{2}}{k T} \cdot \frac{A+B x+\mathrm{Cx}^{2}}{1+D x+\mathrm{Ex}^{2}+\mathrm{Fx}^{3}}
$$

where $x=|J| / k T$. The expression with the two sets of parameters $\mathrm{A}-\mathrm{F}$ is valid for $\mathrm{kT} /|\mathrm{J}|$ greater than 0.5 and $\mathrm{J}$ less or equal to 0 , and as shown in Figure 3, reproduces the calculated magnetic susceptibilities of the 10 membered rings, which should be good approximations for infinite systems for various values of $\alpha$ with an factor $\sum_{n}\left(\left|x_{\text {theo }}-x_{\text {fit }}\right|^{2 / x_{\text {theo }}}{ }^{2}\right)^{1 / 2}$ much better than

0.01 . The error in the alternation parameter and the exchange coupling constant which results from the fit is largely that inherent to the assumptions that the magnetic properties of relatively small rings may be extrapolated to the infinite limit. Presently all of our studies with this model has been carried out in the low field region ( $10 \mathrm{KOe}, 1 \mathrm{kOe}, 100 \mathrm{Oe}$ ); additional studies at higher fields are underway.

MAGNETIC SUSCEPTIBILITY DATA FOR ALTERNATING CHAIN SYSTEMS

The magnetic susceptibility data (11) for catenahexanedionebis (thiosemic arbazato) copper (II), Cu-HTS, in the temperature range $1.8-120 \mathrm{~K}$ are shown in Figuxe 4. The maximum in the magnetic susceptibility curve provides evidence for antiferromagnetic interactions. Attempts to fit the magnetic data by the uniform Heisenberg chain model described above or by the dimer model failed, but an excellent fit was obtained with the alternating chain model. The solid line in Figure 4 was calculated using the Heisenberg alternating chain expression and the magnetic parameters given in Table II. The inability of the uniform chain and dimer models to describe the magnetic behavior is shown by the "best fit" lines which are calculated from these models and are depicted in Figure 4. Data are also provided for the analogous alternating chain catena-octanedionebis (thiosemicarbazato) copper (II), $\mathrm{Cu}$ (OTS).

There are two additional compounds for which an alternating chain model is needed to fit the low temperature magnetic susceptibility data, those being $\mathrm{Cu}\left(4-\right.$ methylpyridine) ${ }_{2} \mathrm{Cl}_{2}$ (32) and $\mathrm{Cu}$ (N-methyl-

imidazole) ${ }_{2} \mathrm{Br}_{2}$. (9) However, both of the compounds have uniformly spaced linear chain structures at room temperature. (33-35) Dielectric measurements on compacted samples of $\mathrm{Cu}$ (4-methy1pyridine) ${ }_{2} \mathrm{Cl}_{2}$ and $\mathrm{Cu}\left(\mathrm{N}-\right.$ methylimidazole) ${ }_{2} \mathrm{Br}_{2}$ pro-

vide evidence for structural phase transformations. As shown in Figure 5, a first order phase transition is clearly indicated by the anomaly in the capacitance data near 50K. There are multiple anomalies in the temperature dependent capacitance data for $\mathrm{Cu}\left(\mathrm{N}\right.$-methylimidazole) ${ }_{2} \mathrm{Br}_{2}$. A first order transition near $105 \mathrm{~K}$ may be a result of a "freezing in" of the expected free rotation of the N-methyl group, and a transition near $50 \mathrm{~K}$ is probably comparable to the alternating chain transformation in $\mathrm{Cu}$ (4-methylpyridine) ${ }_{2} \mathrm{Cl}_{2}$. The magnetic parameters are given in Table II.

The alternating Heisenberg chain model and expressions derived from the cluster approach permit a description of the magnetic susceptibility of the compounds catena-octanedfone- and catena-hexanedionebis (thiosemicarbazato) copper(II), catena-di- $\mu$-chlorobis

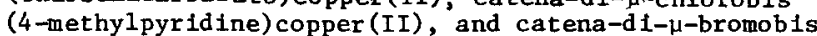

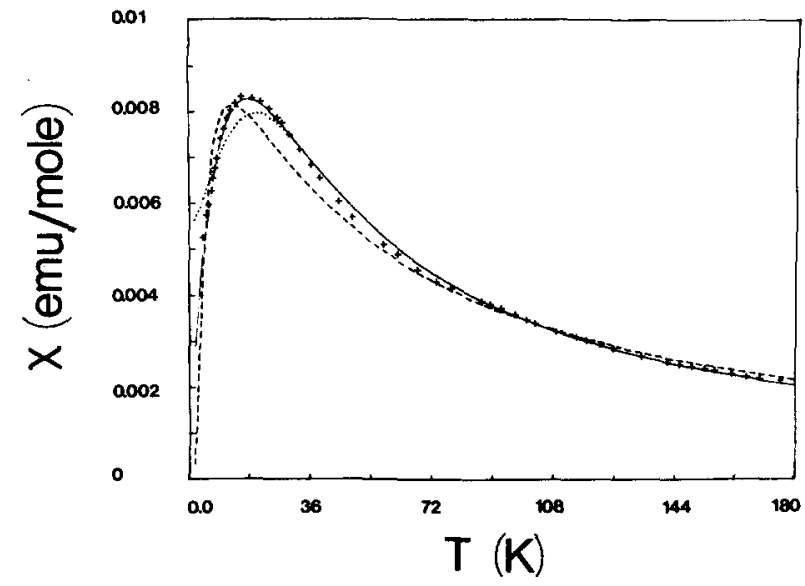

Figure 4. Magnetic susceptibility versus temperature data for catena-hexane-dionebis (thiosemicarbazato) copper(II). The solid line is the best fit of the Heisenberg altemating linear chain model with $J=-20.2 \pm 0.2 \mathrm{~cm}^{-2}, \alpha$ $=0.92 \pm 0.02$, and $g=2.06 \pm 0.02$. For purposes of comparison the "best fits" to the uniform Heisenberg chain $\left(\cdots, J=-20.7 \mathrm{~cm}^{-2}, J^{\prime}=2.76 \mathrm{~cm}^{-2}\right.$, $g=2.22)$ and dimer (--.-, $2 . J=-6.4 \mathrm{~cm}^{-l}$, $g=2.23, \theta=-32.5 \mathrm{~K}$ ) models are also shown.

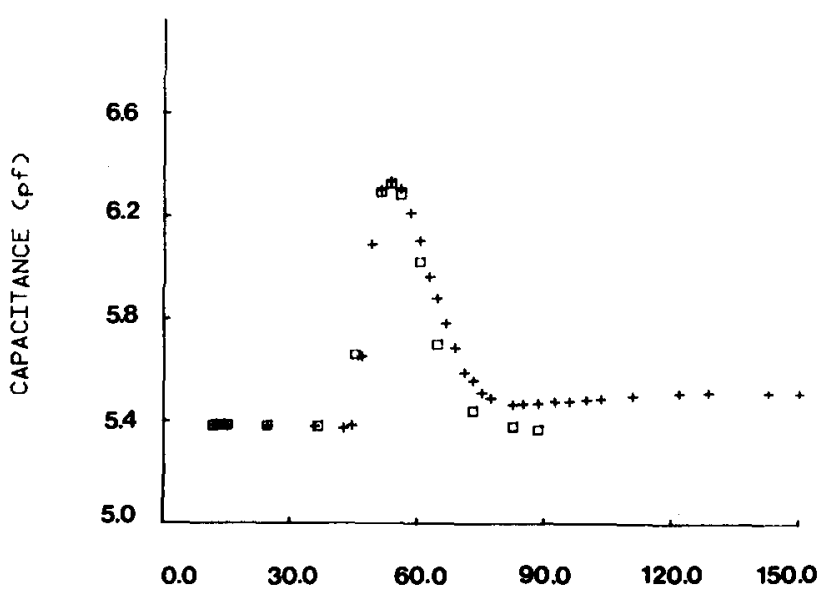

TEMPERATURE (K)

Figure 5. Temperature dependent capacitance data for a pressed pellet sample of actena-diu-chlorobis (4-methylpyridine) copper(II) ( 0 , temperature increasing, + temperature decreasing).

Table II. Magnetic Parameters for the Alternating Cha in Compounds

\begin{tabular}{llll}
\hline Compound & $\mathrm{J}, \mathrm{cm}^{-1}$ & $\alpha$ & $\mathrm{g}$ \\
\hline Cu-HTS & -10.1 & 0.91 & 2.06 \\
$\mathrm{Cu}-\mathrm{OTS}$ & -12.9 & 0.90 & 2.11 \\
$\mathrm{Cu}(4 \mathrm{MP})$ & ${ }_{2} \mathrm{Cl}_{2} \mathrm{a}-9.6$ & 0.67 & 2.17 \\
$\mathrm{Cu}(\mathrm{NMI})$ & ${ }_{2} \mathrm{Br}_{2} \mathrm{~b}-7.2$ & 0.4 & 2.14 \\
\hline
\end{tabular}

$a_{4 M P}=4-$ methylpyridine

$\mathrm{b}_{\mathrm{NMI}}=\mathrm{N}$-methylimidazole 
(N-methylimidazole) copper(II). It is reasonable to suggest that the sulfur bridged compounds have alternating chain structures since the analogous compound $\mathrm{Cu}-\mathrm{KTS}$ has been shown by X-ray diffraction studies (26) to have an alternating chain structure. $X$-ray data for twinned crystals of $\mathrm{Cu}\left(4\right.$-methylpyridine) ${ }_{2} \mathrm{Cl}_{2}$ $(33,34)$ are consistent with a uniform chain structure at room temperature, but an anomaly in the dielectric behavior signals a structural phase transition presumably to an alternating chain as suggested by the low temperature magnetic susceptibility data. Multiple anomalies were seen in the dielectric behavior of $\mathrm{Cu}\left(\mathrm{N}\right.$-methylimidazole) ${ }_{2} \mathrm{Br}_{2}$ and we suggest that a structural phase transition from the uniform chain at room temperature (35) has occurred, thus permitting an explanation of the low temperature magnetic and magnetization data. The neutron diffraction results (36) for $\mathrm{Cu}$ (N-methylimidazole) ${ }_{2} \mathrm{Br}_{2}$ at $4 \mathrm{~K}$ suggest that this problem merits additional attention.

\section{LADDER SYSTEMS}

Ladder systems have also been investigated using the cluster approach. Although it had been thought for a long time that $\mathrm{Cu}\left(\mathrm{NO}_{3}\right)_{2} \cdot 2 \cdot 5 \mathrm{H}_{2} \mathrm{O}$ was an

example of a spin ladder, in view of the ladder-1ike structure at room temperature, (6) recent work has shown that the magnet1c data are consistent with an alternating chain. Weller (15) has carried out calculations for short ladder-1ike chains and rings. For the purposes of illustration, the energy levels resulting from the calculation for a four member chain are plotted in Figure 6 . There are several important points to consider. First, the ground state of the system is a quintet when both exchange coupling constants are positive, a singlet when both exchange coupling constants are negative or when $\mathrm{J}_{12}<0$ and $\mathrm{J}_{13}>0$, and there is a ground state spin crossover at $\alpha=0.3$ when $J_{12}>0$ and $J_{13}<0$. It is clear that a wide variety of behavior is to be expected from ladder compounds and this expectation provides the stimulation for extensive synthetic and structural studies.

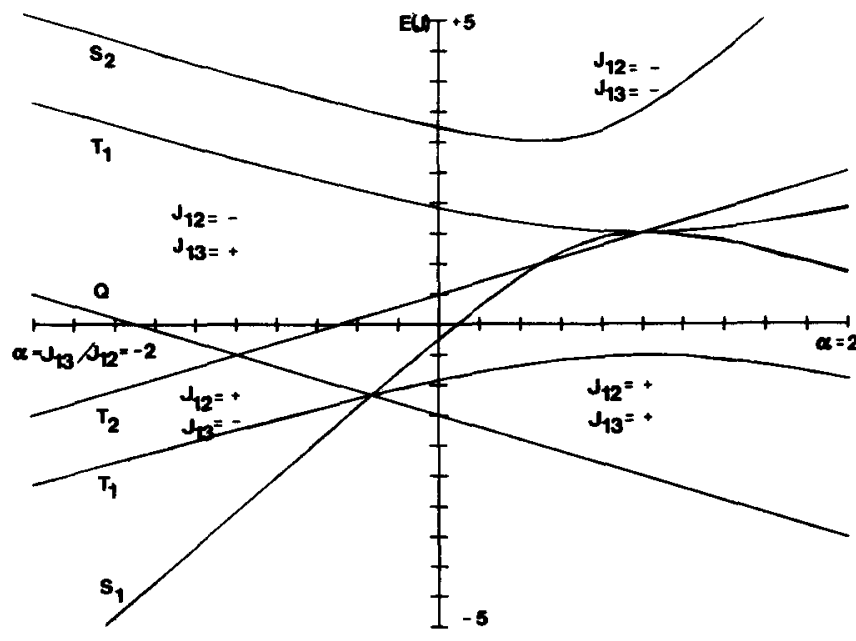

Figure 6. Energy level diagram for a spin ladder with four copper(II) ions.

MAGNETIC DATA FOR THE LADDER CATENA-TRICHLOROHYDRAZ INUIMCOPPER (II)

The structure and magnetic properties of (HzH) $\mathrm{CuCl}_{3}$ have been reported by Brown, et al. (14) The ladder-like structure of the compound is shown in Figure 7. The coordination about the copper ion is the familiar $4+2$ form with the equitorial plane being

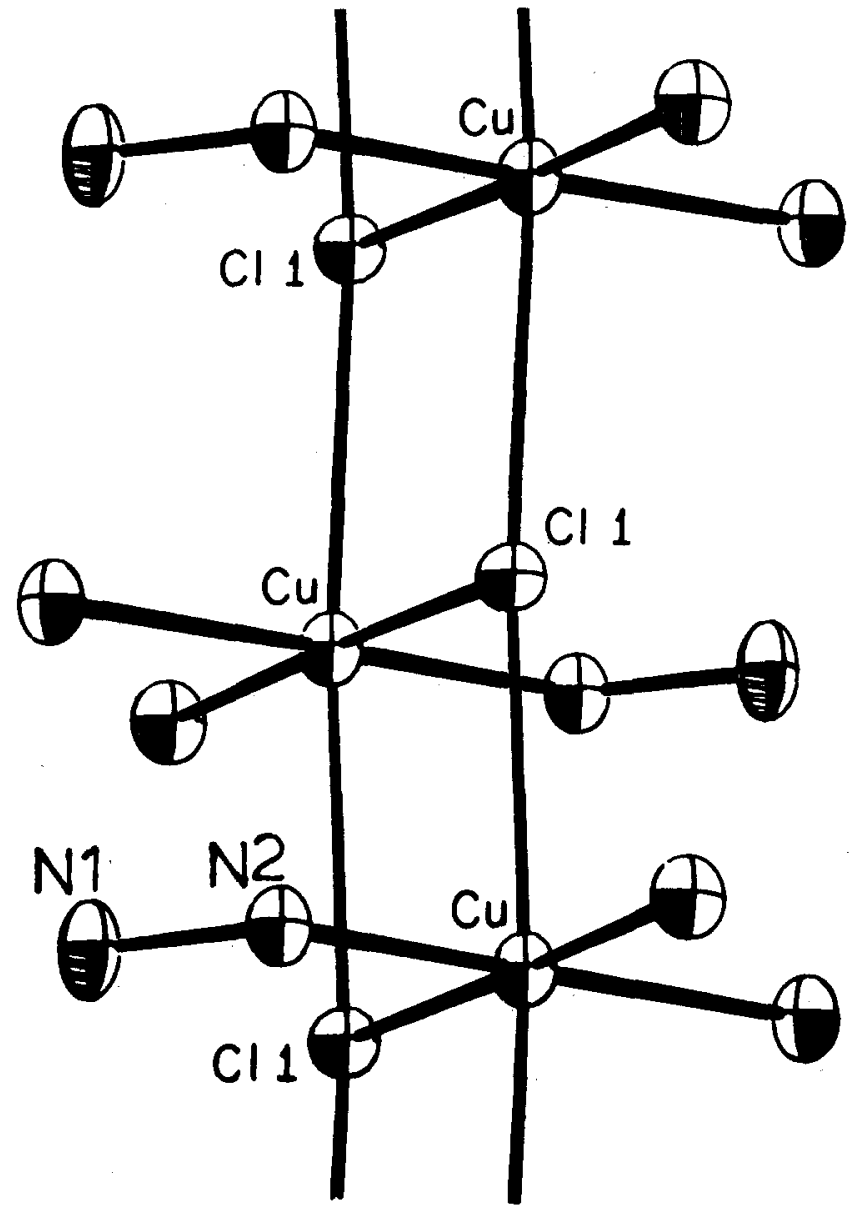

Figure 7. The structure of the Zadder compound hydrazinium copper(II) chloride.

formed by three chloride fons and the monodentate hydrazinlum ion. The apical positions in the coordination sphere are occupied by chloride ions from adjacent units. The Cu-Cl in-plane bridging distance is 2.297(1) $\dot{A}$ and the out-of-plane bridging distance is $2.856 \AA$

The magnetic susceptibility data reflect an antiferromagnetic interaction with a maximum in the magnet ic susceptibility at about $12 \mathrm{~K}$. The data above $25 \mathrm{~K}$ may be $f$ it by the Curie-Weiss law to yield a $g$ value of 2.196, while the entire data set may be fit by an eight membered chain to yield the same $\mathrm{g}$ value, $\mathrm{J}_{12}$ (the $90^{\circ}$ interaction) of $-7.5 \mathrm{~cm}^{-1}$, and $\mathrm{J}_{13}$ (the $180^{\circ}$ interaction) of $-7.0 \mathrm{~cm}^{-1}$. The agreement factor $\mathrm{R}=$ $\left[\Sigma\left(x^{\text {obs }}-x^{\text {calc }}\right)^{2} / \Sigma\left(x^{\text {obs }}\right)^{2}\right]^{1 / 2}$ for the fit is 0.018 . A similiar calculation was carried out for an eight membered ring to yield $\mathrm{J}_{12}=-5.4 \mathrm{~cm}^{-1}, \mathrm{~J}_{13}=$ $-5.3 \mathrm{~cm}^{-1}, \mathrm{~g}=2.14$, and $\mathrm{R}=0.013$. The calculations firmly support the conclusion that the superexchange interactions are antiferromagnetic by both exchange pathways, but high field magnetization measurements will have to be made before the magnitude of the coupling constants can be known with certainty.

\section{CONCLUSION}

The cluster approach to the problem of exchange interactions has been reasonably successful in accounting for the magnetic behavior of extended linear chains, alternatingly spaced linear chains, and ladders. With use of the cluster approach it has been posstble to define exactly the indivi- 
dual exchange interactions and obtain detalled analyses of magnetic properties. There is a problem in that small clusters which are not realistic models may yield fits to experimental data which are nearly as good as the fits with larger clusters, but the magnetic parameters are usually unacceptable. As research in this area progresses, an adequate body of data will be generated so that reasoned judgements may be made about the acceptability of magnetic parameters from model calculations.

\section{ACKNOWLEDGEMENTS}

This research was supported in part by the office of Naval Research and by the National Sclence Foundation through grant CHE80 09685. We wish to thank Professor $\mathrm{J}$. Reedijk for sending us the plot of magnetic susceptibility data for $\mathrm{Cu}$ (N-methylimidazole) ${ }_{2} \mathrm{Br}_{2}$, and $\mathrm{Dr}$. S. Mitra for a preprint of the work on $\left[\mathrm{Cu}(\alpha-d-n-b t c){ }_{2}\right]_{2}$.

\section{REFERENCES}

1. J.C. Bonner and M.E. F1sher, Phys. Rey. [Sect. A] $1964,135,640$.

2. C.-Y. Weng, Ph.D. Dissertation, Carnegle-Melion University, Pittsburgh, PA, (1968).

3. J.C. Bonner, S.A. Friedberg, H. Kobayash1, B.E. Meyers. Proceedings of the Twelfth International Conference on Low Temperature Physics, Kyoto, Japan, 1970, edited by E. Kanda (Kelgaku, Tokyo, 1971), p. 691 .

4. J.C. Bonner and S.A. Frledberg. Proceedings of the International Conference on Phase Transitions and Their Applications in Material Sclence, edited by Hanish, H.K., Roy, R., Cross, L.E. Pergamon Press, New York, (1973), p. 429.

5. K.M. Diederix, H.W.J. Blote, J.P. Groen, T.O. Kiaasen, N.J. Poulis. Phys. Rev. B (1979), 19, 420.

6. B. Morosin. Acta Crysta1logr. B (1970), 26, 1203.

7. J.S. Valentine, A.J. Silverstein, Z.G. Soos, J. Amer. Chem. Soc. (1974), 96, 97.

8. J.W. Bray, H.R. Hart, L.W. Interrante, I.S. Jacobs, J.S. Kasper, G.D. Watkins, S.H. Wee, J.C. Bonner. Phys. Rev. Lett. (1975), 35, 744.

9. J.J. Smit, L.J. DeJongh, J.A.C. van Doijen, J. Reedijk, J.C. Bonner. Physica B (1979), 97, 229.

10. L.E. Warren, S.M. Horner, W.E. Hatfield. J. Amer. Chem. Soc. (1972), 94, 6392.

11. J.W. Ha1l, W.E. Marsh, R.R. Weller, W.E. Hatfield. Inorg. Chem, in press.

12. W. Duffy and K.P. Barr. Phys. Rev. (1968), 165, 647.

13. H.M. Helis, W.H. Goodman, R.B. Wilson, J.A. Morgan, D.J. Hodgson. Inorg. Chem. (1977), 16, 2412.
14. D.B. Brown, J.A. Donner, J.W. Hall, S.R. Wilson, D.J. Hodgson, W.E. Hatfield. Inorg. Chem. (1979), $18,2635$.

15. R.R. Weller, Ph.D. Dissertation, University of North Carolina, Chapel Hill, NC 27514, (1980).

16. J.W. Ha11, Ph.D. Dissertation, University of North Carolina, Chapel Hill, NC 27514, (1977).

17. J.C. Bonner, Ph.D. Dissertation, University of London, (1968).

18. W.E. Hatfield, R.R. Weller, J.W. Hall. Inorg. Chem. in press.

19. F.W.B. Einstein and J.S. Field. Acta Crystallogr. $1074, B 30,2928$.

19a. See, for example, J.W. Stout and R.C. Chisholm, J. Chem. Phys. (1962), 36, 979.

20. J.F. Villa and W.E. Hatfield. Inorg. Chem. (1971), 10,2038 .

21. M. Bonnamico, G. Dessy, A. Mugnoli, A. Vaciago L. Zambonel11. Acta Crystallogr . (1965), 19, 886 .

22. S.A. Al'tshuler, R. Kirmse, B.V. Solov'ev. J. Phys. C: Solid State Phys. (1975), 8, 1907.

23. W.E. Hatfield, H.W. Richardson, J.R. Wasson. Inorg. Nuc1. Chem. Letters, (1977), 13, 137.

24. A.M. Landredi, A. Tiripicchio, M.T. Came11 ini. J. Chem. Soc. Dalton Trans. (1975), 2168.

25. W.E. Blumberg and J. Peisach. J. Chem. Phys. (1968), 49, 1793

26. M.R. Taylor, J.P. Glusker, E.J. Gabe, J.A. Minkin. Bio inorg. Chem. (1974), 3, 189.

27. P.D.W. Boyd, S. Mitra, C.L. Raston, A.H. White. $\mathrm{J}$. Chem. Soc. Dalton Trans. submitted.

28. V.H. Crawford, H.W. Richardson, J.R. Wasson; D.J. Hodgson, W.E. Hatfield. Inorg. Chem. (1976), 15, 2107.

29. S.G.N. Roundhill, D.M. Roundhill, D.R. Blomqulst, C. Landee; R.D. Willett, D.M. Dooley; H.B. Gray. Inorg, Chem. (1979), 18,831.

30. W.E. Estes, W.E. Hatfield, J.A.C. van ooijen, J. Reedijk. J. Chem. Soc. Dalton Trans., in press.

30a. J.C. Bonner, H.W.J. Blöte, J.W. Bray, I.S. Jacobs, J. Appl. Phys. (1979), 50, 1810 .

31. A.K. Gregson, Aust. J. Chem., in press.

32. V.H. Crawford and W.E. Hatfield. Inorg. Chem. (1977), 16, 1336.

33. W.E. Marsh, E.J. Valente, D.J. Hodgson, W.E. Hatfield, unpublished observations.

34. J.A.C. van Ooijen, J. Reedijk, A.L. Spek. J. Chem. Soc. Dalton Trans. (1979), 1183.

35. J.C. Jansen, H. van Koningsveld, J.A.C. van Ooijen. Cryst. Struct. Commun. (1978), ㄱ, 637.

36. D.W.J. Ydo, J. Reedijk, private communication, have determined that the space group at $4.2 \mathrm{~K}$ is the same as that at $300 \mathrm{~K}$. 